Audit on clozapine dose and plasma level correlation for patients with chronic treatment-resistant psychosis

Olivia Macnamara ${ }^{\star}$ John Lawton and Sudheer Lankappa

Nottinghamshire Healthcare NHS Foundation Trust

${ }^{*}$ Corresponding author.

doi: 10.1192/bjo.2021.273

Aims. Clozapine is associated with a risk of severe adverse events for which there are current monitoring systems are in place; however, there are no established regimens for monitoring of clozapine plasma levels. Recent Medicines and Healthcare products Regulatory Agency (MHRA) guidance advises clozapine levels should be monitored in certain clinical situations where toxicity may be suspected. This audit aimed to evaluate current practice of clozapine level monitoring within one Local Mental Health Team (LMHT).

Method. Electronic (RiO) records of 41 patients (33 male, 8 female; aged from 27 to 76 years; mean age 45 years) registered to the ZTAS system within the Nottingham City Central LMHT were reviewed. $46 \%$ had been on clozapine for over 16 years. $73.3 \%$ of patients were within clusters 12 and 13;25.4\% of patients were in cluster 11 , with one patient in cluster 8 . Dates of clozapine plasma level tests for each patient between 2006 and 2020 were found on the electronic NoTIS system, along with clozapine, norclozapine and total clozapine levels. Concurrent clozapine dose and regimens were obtained from pharmacy records from 2018 onwards.

Result. 273 clozapine plasma levels were conducted between 2006 and 2020. The average interval between levels taken was 10 months, 2 weeks but had a wide range, the shortest interval being 2 days, the longest being 13 years. 88 levels taken were $>600 \mathrm{ug} / \mathrm{L}$, suggesting increased toxicity risk. 108 levels were $<350 \mathrm{ug} / \mathrm{L}$, suggesting possible sub-optimal dosing or noncompliance. Statistical tests on correlation coefficient, although statistically non-significant $(\mathrm{R}=0.37)$, showed a positive trend between total clozapine dose and the plasma level between all 3 parameters (i.e. clozapine, norclozapine and total clozapine).

Conclusion. There does not appear to be any routine plasma clozapine level monitoring throughout the LMHT with an average interval between tests of 10 months. There was a non-significant but positive trend between total daily dose of clozapine and clozapine level. $32 \%$ of clozapine levels returned were higher than the recommended level. We would recommend as suggested in the guidelines from MHRA, clozapine plasma levels should be monitored in certain clinical situations with increased toxicity risk. Trough levels should be taken with records of time of previous dose taken. Limitations of this study included a small sample size (41 patients) with data collection reliant on electronic systems. It was unclear if these results represent trough levels, making values difficult to interpret. Multifactorial impact on clozapine metabolism causes wide patient variability in plasma levels.

\section{The diagnosis and management of adult ADHD in HMP Elmley, a Category B remand prison}

Kathleen McCurdy* and Nosa Igbinomwanhia

Oxleas NHS Foundation Trust, London

${ }^{\star}$ Corresponding author.

doi: 10.1192/bjo.2021.274

Aims. Attention deficit hyperactivity disorder (ADHD) is a highly prevalent disorder in young adult prisoners. This audit aimed to identify how many residents are prescribed medication treatment for ADHD in HMP Elmley and whether those seen by the prison psychiatrists have been managed in line with
NICE guidelines. We also audited waiting times and time to follow-up appointments. This was done with the overall aim to identify potential areas for development.

Method. We performed a spot audit of all residents in HMP Elmley who were prescribed ADHD medication on 4th November 2019, using their electronic patient records. Appointments with the psychiatrists were then subdivided into initial assessments and follow-up appointments for the purpose of analysis. Performance was measured against NICE Guideline [NG87]: Attention deficit hyperactivity disorder: diagnosis and management. We also calculated the waiting times for initial appointment and follow-up appointment.

Result. We found that 33 of residents were on ADHD medication at the time of the audit, approximately $3 \%$ of the prison population. $64 \%$ of those had a pre-existing diagnosis and $36 \%$ had been given a new diagnosis at HMP Elmley. Of those newly diagnosed $100 \%$ had undergone a Diagnostic Interview for Adults in ADHD (DIVA) assessment for diagnosis.

Baseline physical health checks had been performed in $68 \%$ of patients prior to starting medication and a cardiovascular examination had occurred in 9\%. At follow-up 100\% of patients had their physical observations and weight checked and their symptoms reviewed.

$91 \%$ of patients were started on methylphenidate or lisdexamfetamine as first line treatment, with the rest started on atomoxetine and the reason for this documented.

$100 \%$ patients were offered general psychological support.

There was a mean 22 day wait for an initial appointment (range 0-65) and a mean 20 day wait from starting medication to a psychiatric follow-up appointment (range 8-37)

Conclusion. The number of residents treated for ADHD in HMP Elmley is relatively low (3\%) compared to the estimated prevalence in prison population.

The key areas for improvement are in baseline cardiovascular examinations and physical health evaluations. The waiting time between initial psychiatric appointment and follow-up is another area where improvement is needed and this will form the basis of a quality improvement project.

Future steps include setting up a specific ADHD clinic with an allocated nurse practitioner to support, producing a template for ADHD assessments and follow-ups, producing a local policy on ADHD and developing specific resources for ADHD psychoeducation.

\section{Escalation of care planning on an older adult inpatient unit during the COVID-19 pandemic}

\author{
Alexander McDermott* and Jennifer Rankin \\ Angelton Clinic - Cwm Taf Morgannwg University Healthboard \\ ${ }^{*}$ Corresponding author.
}

doi: 10.1192/bjo.2021.275

Aims. Initial planning during the first wave of the COVID-19 pandemic involved difficult decision making for many clinicians. The Older Adult Mental Health Wards in Bridgend were relocated from the district general hospital (Princess of Wales) and merged at Angelton Clinic, an off site separate unit. It was therefore essential that patients had clear escalation of care plans as access to medical input was limited and transfer to hospital potentially not appropriate in the later stages of chronic illness such as dementia.

The initial aim of the PDSA cycle was to assess the level of compliance with Do Not Attempt Resuscitation (DNAR) discussions and if appropriate, DNAR documentation. The other aim was to assess the utilisation of Escalation of Care plans. 
Method. An audit of patients MDT medical notes on 38 admitted to Angelton clinic was carrired out in March. It was documented if the patient had a clear DNAR or Escalation plan that was easily accessible in the front of the notes. The guidelines compared to were the GMC recommendations that patients 12 months of should have a discussion about risks and benefits associated with Cardiopulmonary Resuscitation. If the patient lacks capacity a best interest decision should be made with nearest relatives. Discussions should also be had with patients and family in in regards to and transfer to a medical ward.

Upon completion of the initial PDSA cycle, views were sought from the wider MDT a new escalation of care proforma was designed. This was implemented by education and communication with members of the medical team. This was to be clearly placed in the notes, with the DNAR form if that was appropriate. Result. All inpatient notes were audited at Angelton Clinic in March 2020. It was found that only $18 \%$ of patients had Escalation of Care plans in comparison to $84 \%$ of notes which had DNAR forms. Previous escalation of care forms were not being utilised appropriately.

Upon implementation of the Escalation of Care proforma, a re-audit of the audit cyle was completed. In July 2020 it was found that $78 \%$ of notes had completed Escalation of Care forms with $83 \%$ had completed DNAR forms.

Conclusion. To enable ongoing sustained improvement, the unit Nurse Practitioner will champion its completion. The audit findings have been shared with the newly rotated junior doctors and proformas were made available on all inpatient wards.

You have to acknowledge the problem before you can address the problem: Audit looking at identification of co-existing substance misuse in a Liaison Psychiatry patient population

Emma McLean ${ }^{1 \star}$, Akash Kadiwar ${ }^{2}$ and Mariam Alexander ${ }^{3}$

${ }^{1}$ Imperial College Healthcare NHS Trust; ${ }^{2}$ Northampton General Hospital NHS Trust and ${ }^{3}$ West London NHS Trust

${ }^{\star}$ Corresponding author.

doi: 10.1192/bjo.2021.276

Aims. To evaluate if patients referred to Ealing Liaison Psychiatry Service (ELPS) with co-existing substance use are being appropriately identified as per NICE guidelines.

Patients with co-existing substance misuse have greater morbidity and mortality and it is therefore important to identify these patients to optimise their management. NICE recommends that all patients are asked about their substance use.

Anecdotally, our team felt we were doing a good job of identifying and managing such patients but we had no objective evidence of this.

Method. Completed a retrospective audit looking at a sample of patients referred to ELPS over two weeks in December 2019.

A training session for ELPS was then held to highlight the initial audit results and NICE guideline recommendations.

We then repeated the audit over two weeks in March 2020.

Result. Initial audit (100 patients):

Only $69 \%$ of patients asked about substance use. From those asked, 50-65.2\% were using a substance, most commonly alcohol.

None of the patients over the age of 80 were asked about substance use vs $79.5 \%$ of patients aged $20-40$ years.

$55 \%$ of females vs $81 \%$ of males were asked about illicit substances.

$33.3 \%$ of ward referrals vs $74.2 \%$ of Emergency Department referrals asked about substance use
Re-audit (53 patients):

Significant improvement across all areas

93\% now asked about substance use

$60 \%$ of over 80 s, $96 \%$ of females and $85 \%$ of ward referrals were now being correctly asked about substance use

Conclusion. We were surprised to find that we were initially not meeting NICE standards regarding asking patients about their substance use.

Acknowledging this problem during our training session proved to be effective.

This knowledge will help us develop our care pathways with our Acute colleagues and the Drug and Alcohol Liaison Service.

\section{Antipsychotic prescribing in dementia}

James McLoughlin ${ }^{1 *}$, Emma Roemmele ${ }^{2}$, Marguerite Cryan ${ }^{2}$, Catherine Dolan ${ }^{2}$ and Geraldine McCarthy ${ }^{2}$

${ }^{1}$ Psychiatry Department, University Hospital Galway and ${ }^{2}$ Sligo

University Hospital

${ }^{\star}$ Corresponding author.

doi: 10.1192/bjo.2021.277

Aims. The majority of people with dementia will develop one or more behavioural or psychological symptoms of dementia (BPSD) as the illness progresses. Treating these symptoms in diverse residential environments is a challenge, with frequent prescribing of antipsychotic medications. The risks and limited benefits of antipsychotic use in this context are well recognised, prompting national guidelines in Ireland to improve prescribing patterns.

1) Assess the frequency and appropriateness of prescribing of antipsychotic medication in older adults with BPSD referred to Psychiatry of Old Age service in the West of Ireland (Sligo) by comparing with best practice guidelines.

2) Address identified deficits via quality improvement initiatives within department.

Method. Audit standards were set using draft National Clinical Guidelines and NICE guidelines for prescribing in dementia to develop a study specific audit tool.

Items assessed included: the frequency of review of antipsychotic use, whether or not non-pharmacological methods were trialled, if there was an assessment of benefit of the antipsychotic and discussion or risks, if a reduction/discontinuation of antipsychotic was considered, if metabolic monitoring was achieved.

Clinical records for all patients actively under the care of the clinical team with a diagnosis of BPSD were assessed using this tool at the time of the study.

Result. 49 patients with BPSD were attending the service in this time period. $58 \%(n=29)$ of the entire cohort were prescribed an antipsychotic, most commonly quetiapine. Patients cared for at home showed the lowest levels of antipsychotic use at 50\% $(\mathrm{n}=18)$, while those who were in nursing home $(80 \%, n=8)$ and hospital care $(100 \%, \mathrm{n}=3)$ showed higher rates, though this sample size was too small to demonstrate statistical significance, $\chi^{2}=5.12 \mathrm{p}=0.077$.

Exploration of non pharmacological management of BPSD, documentation of discussion of risks of AP medication (metabolic, cardiovascular, falls, sedation, extrapyramidal), attempt at dose reduction or antipsychotic withdrawal were all achieved in less than $45 \%$ of cases (range $33-45 \%$ ).

Conclusion. This audit revealed higher than expected rates of antipsychotic prescribing in our BPSD cohort. It also revealed suboptimal documentation around the use of antipsychotics in this population during clinical interactions. 